\title{
The breast pain clinic: a rational approach to classification and treatment of breast pain
}

\author{
C.D.M. Griffith, C.S. Dowle, C.P. Hinton and R.W. Blamey \\ University Department of Surgery, City Hospital, Nottingham, UK.
}

\begin{abstract}
Summary: Three hundred and fifty women complaining of breast pain symptoms of sufficient severity to interfere with their normal lifestyle were reviewed in a special breast pain clinic over a 5 year period.

Seventy-two patients (21\%) had spontaneous resolution of breast pain and they required reassurance only before discharge. Of the remaining 278 patients, accurate classification of breast pain syndromes was achieved in $89 \%$, the commonest syndrome being cyclical breast pain which accounted for $54 \%$ of the women followed up. The remaining womens' breast pain was classified as trigger zone (14\%), continuous $(8 \%)$, Tietze's disease (5\%), spinal root $(4 \%)$, duct ectasia (4\%) and psychological depression (2\%). In the remaining 25 patients ( $9 \%$ ) the breast pain could not be classified.

The experience from this clinic is that a majority of women complaining of severe breast pain symptoms can be accurately classified and appropriate therapy instituted.
\end{abstract}

\section{Introduction}

Breast pain is the commonest reason for a woman with breast related symptoms to consult her general practitioner. ${ }^{1}$ Preece et al. ${ }^{2}$ classified breast pain in women and demonstrated several distinct syndromes on which Bishop \& Blamey ${ }^{8}$ from the Nottingham unit later exanded.

In this paper we describe our experience in the breast pain clinic at the City Hospital, Nottingham, in the classification and treatment of 350 women selected from the general breast clinic with severe breast pain symptoms.

\section{Patients and methods}

Over 1,500 patients with breast related symptoms are referred annually by general practitioners to the breast clinic in the Professorial Surgical Department. ${ }^{3}$ Although one third of these patients will have breast pain as a symptom, only about 100 patients per year have symptoms bad enough to require investigation and treatment in the breast pain clinic. Obvious exclusions are patients whose pain is related to a clinically apparent carcinoma, cyst or abscess and those patients whose breast pain is relatively mild and does not

Correspondence: C.D.M. Griffith, M.D., F.R.C.S. (Ed), Queen's Medical Centre, Nottingham NG7 2UH, UK.

Accepted: 20 January 1987 interfere wtih their normal lifestyle.

When a patient attends the breast clinic, her symptoms are recorded, a careful examination of the breasts is carried out and mammograms obtained in women over 40 years of age when indicated if suspicious areas of breast tissue are found. If no dominant lump is found, the breast pain is classified and appropriate therapy started. Patients are reviewed on a regular basis and a subjective assessment of response to treatment made by the examining doctor.

\section{Classification of breast pain symptoms}

Of the total of 350 patients reviewed in the breast pain clinic, 72 were discharged after further examination and reassurance without treatment because of spontaneous resolution of symptoms. In the remaining group of 278 patients, classification of breast pain was achieved in 253 patients (Table I).

Cyclical breast pain was typically poorly localized and bilateral in most women but could be unilateral. It was clearly related to the menstrual cycle with exacerbations in the premenstrual phase and pain relief during menstruation.

A trigger zone was present when the patient complained of a well localized pain at a consistent site in the breast accurately identified on 2 or more visits to the clinic. Continuous breast pain was characterized 
by burning or aching of both breasts with tenderness which did not vary during the menstrual cycle. In Tietze's disease, the breast pain was located to the inner quadrants of the breast and was continuous in nature with the clinical finding of tenderness over one or more costochondral junctions, usually unilateral.

A diagnosis of spinal root syndrome was made when patients complained of pain radiating from the breast to the neck and outer border of the ipsilateral arm with associated limitation of movement of the cervical spine. This syndrome was often associated with the paraesthesia on the affected side and with X-ray changes of degenerative disease. Patients with persistent burning pain situated behind the nipple or nipples associated with palpable ectatic ducts with green or yellow stained nipple discharge were classified as duct ectasia.

In 25 patients the breast pain could not be accurately classifed. These patients were regularly reviewed to ensure no treatable breast pain syndrome emerged, and then discharged. A further 4 patients were clinically depressed with the complaint of breast pain as a primary symptom. Pregnancy as a cause of breast pain must be excluded since it is an absolute contraindication to the use of most of the drugs commonly prescribed in the treatment of breast pain.

\section{Results of treatment}

Most patients with continuing breast pain symptoms improved following treatment instituted at the breast pain clinic (Table II).

The largest group of patients were those complaining of cyclical breast pain. Treatment with the antigonadotrophin danazol was effective in $66 \%$ but 20 women required up to three separate three month courses of the drug to obtain relief. In 24 women the drug was withdrawn because of severe side effects such as headache, weight gain, or muscle cramps. Forty-six women who did not respond to danazol or suffered side effects were treated with alternative agents such as
Table I Classification of breast pain symptoms in 278 patients

\begin{tabular}{lrr}
\hline & Number & $\%$ \\
\hline Cyclical & 150 & 54 \\
Trigger zone & 39 & 14 \\
Continuous & 22 & 8 \\
Tietze's disease & 14 & 5 \\
Spinal root & 11 & 4 \\
Duct ectasia & 11 & 4 \\
Depression & 4 & 2 \\
Unclassified & 25 & 9 \\
\hline
\end{tabular}

the diuretic cyclopenthazide (27) or hormonal manipulation by bromocriptine (10), norethisterone (4) or os tamoxifen (4). In five patients clinical response was obtained using evening primrose oil after all other $\perp$ treatment had failed.

Treatment was difficult in the 24 patients with $\doteq$ continuous breast pain; however nine patients respon- $O$ ded to the use of danazol. Symptomatic treatment with simple analgesics was marginally effective in the $c$ remainder; however, in four patients the pain was sufficiently severe and intractable for them to request $\vec{\bullet}$ subcutaneous mastectomy with prosthetic implang $\stackrel{\infty}{\checkmark}$ Two of these patients, however, still had some pain. following mastectomy.

Of the 38 women with trigger zone breast pain, 20 patients responded to simple measures such as a firm supportive bra worn night and day plus simple analgesics as required. A further 13 women were successfully treated by surgical excision of the tender area and five others responded to local injections of hydrocortisone with lignocaine.

Tietze's disease was usually a self-limiting condition and often improved spontaneously, but four of the 14 patients with this condition were helped by injections of lignocaine and methylprednisolone into the affected costochondral junctions.

Most of the 12 patients with spinal root syndrome

Table II Treatment resulting in relief of symptoms of breast pain

\begin{tabular}{|c|c|c|c|c|c|c|}
\hline \multirow[b]{2}{*}{ Treatment } & \multicolumn{6}{|c|}{ Type of breast pain } \\
\hline & $\begin{array}{l}\text { Cyclical } \\
n=150\end{array}$ & $\begin{array}{c}\text { Trigger zone } \\
n=38\end{array}$ & $\begin{array}{c}\text { Continuous } \\
n=24\end{array}$ & $\begin{array}{l}\text { Tietze's } \\
n=14\end{array}$ & $\begin{array}{c}\text { Spinal root } \\
n=12\end{array}$ & $\begin{array}{c}\text { Duct ectasia } \\
n=11\end{array}$ \\
\hline Danazol & 100 & 0 & 9 & 0 & 0 & 0 \\
\hline Diuretic & 27 & 0 & 0 & 0 & 0 & 0 \\
\hline Hormonal manipulation & 18 & 0 & 0 & 0 & 0 & 0 \\
\hline Efamol (Primrose oil) & 5 & 0 & 0 & 0 & 0 & 0 \\
\hline Local steroids & 0 & 5 & 0 & 4 & 0 & 0 \\
\hline Support bra and analgesics & 0 & 20 & 11 & 0 & 0 & 8 \\
\hline Physiotherapy & 0 & 0 & 0 & 0 & 9 & 0 \\
\hline Surgery & $\mathbf{0}$ & 13 & 2 & 0 & 0 & 3 \\
\hline
\end{tabular}


were effectively treated by a soft cervical collar and physiotherapy, including shoulder girdle exercises which the patient carried out at home after instruction by the physiotherapist.

The majority of patients with pain due to duct ectasia responded to simple analgesics and local support but in three patients the pain was sufficiently severe to warrant excision of the major duct system by a Hadfield's procedure. ${ }^{4}$ All four patients who were ultimately diagnosed as suffering from depression manifesting as breast pain had a previous history of psychiatric disturbance and were referred for psychiatric help.

\section{Discussion}

Despite the fact that breast pain is a common complaint, many doctors find the pain difficult to treat and may regard it as trivial and unworthy of treatment. Breast pain may be seen as a symptom of neurosis although the psychoneurotic profile of women with breast pain has been demonstrated as being no different from women with a recognized surgical condition such as varicose veins. ${ }^{6}$

Women are often anxious that breast pain heralds serious disease of the breast, particularly cancer. In the majority the discomfort is not severe and clinical examination and confident reassurance is frequently all that is required. In some patients the pain is of sufficient magnitude to make the patient irritable and bad tempered which may affect her relationship with her spouse and family or interfere with her performance at work. ${ }^{7}$ In this group requiring treatment, accurate classification of breast pain symptoms is the

\section{References}

1. Nichols, S., Waters, W.E. \& Wheeler, M.J. Management of female breast disease by Southampton general practitioners. Br Med J 1980, 2: 1450-1453.

2. Preece, P.E., Hughes, L.E., Mansel, R.E., Baum, M., Bolton, P.M. \& Gravelle, I.H. Clinical syndromes of mastalgia. Lancet 1976, ii: 670-673.

3. Mikulin, T., Blamey, R.W., Hinton, C.P. \& Holliday, H.W. The spectrum of breast disease: an audit of 1,509 patients attending a breast clinic over one year. National Conference on Benign Breast Disease, Southampton 1985.

4. Hadfield, G.J. The pathological lesions underlying dis- key to successful treatment ${ }^{8}$ and our experience is that this can be achieved in almost $90 \%$ of patients referred to a special breast pain clinic. Breast pain can be the presenting symptom of breast cancer but none of the 350 women referred to the breast pain clinic was subsequently found to have a tumour.

The majority of women with breast pain whose symptoms are severe enough to require treatment will have cyclical breast pain. The aetiology of this condition is unknown but its clear relationship to the menstrual cycle has pointed to the female sex hormones as being responsible with perhaps an altered sensitivity of the breast as the target organ. The antigonadotrophin danazol, which also has androgenic properties, was effective in $66 \%$ of our patients with this condition whilst the remainder responded to alternative agents.

Surgery may be required for some patients whose breast pain is related to a trigger zone or duct ectasia where local excision is often effective. A few women with untreatable continuous breast pain may require subcutaneous mastectomy to relieve their symptoms.

Spinal root syndrome and Tietze's disease are treated with conventional measures such as physiotherapy or local steroid injections respectively. Patients whose breast pain is a manifestation of psychiatric disturbance should be given appropriate help.

Our experience with women seen in the breast pain clinic has shown that some have symptoms which are far from trivial. Those with breast pain severe enough to affect their lifestyle and interpersonal relationships will usually have classifiable and treatable pain. Accurate classification of breast pain symptoms can be achieved and this leads to effective therapy for most patients.

charges from the nipple in women. Ann R Coll Surg of Engl 1969, 44: 323-333.

5. Patey, D.H. Two common non-malignant conditions of the breast. Br Med J 1949, 1: 96-99.

6. Preece, P.E., Manse, R.E. \& Hughes, L.E. Mastalgia: psychoneurosis or organic disease. $\mathrm{Br}$ Med J 1978, 1: 2930.

7. Preece, P.E. Mastalgia. The Practitioner 1982, 226: 1373-1384.

8. Bishop, H.M. \& Blamey, R.W. A suggested classification of breast pain. Postgrad Med J 1979, 55: 59-60. 